\title{
An Effective Modulus Predictive Method of POSS Reinforced Resin Matrix Composites
}

\author{
Huazhen Wei, Danyong Wang, Juncong Liu, Yiwei Chen and Shuhu Li \\ Shandong Non-metallic Materials Institute, Jinan 250031, China
}

\begin{abstract}
Compatibility between POSS and polymer was the key element of effecting whether POSS as nanofillers could improve mechanical properties of polymer. Based on really materials and applied manufacturing process, a new kind of an effective modulus predictive method for POSS reinforced resin matrix composites which could compute and analyze modulus of any content ratio of POSS in composites with only one content ratio was built in order to provide theoretical foundations for design and property of POSS/resin composites. According to practically different materials system and corresponding manufacturing processes, few experiments which only needed elastic modulus of one random content-ratio POSS/resin composites were made to predict effective elastic modulus of any other content ratio of POSS/resin composites under the same manufacturing processes by obtaining experimental constants based on numerical progressive iterative approximation method and compute the above experimental constants as basic materials parameters so as to build the whole analysis model in the next step. An excellent agreement was found between data obtained from this study and the experiment.
\end{abstract}

Keywords-posst; composites; predictive method

\section{INTRODUCTION}

Kinds of modification techniques had been used for resin matrix composites in order to improve mechanical properties of resin matrix composites in recent years. Polyhedral oligomeric silsesquioxanes (POSS) as a new kind of nano-filler with nano-scale and cage-like structure organic-inorganic hybrid compound could be added into polymer to improve anti-thermal, anti-oxidation, surface hardness and mechanical properties which has aroused great attentions of numerous scientists[1-7].

The excellent and precise control of POSS content ratio in resin matrix composites and corresponding surface compatibility during manufacturing POSS reinforced resin matrix composites could greatly improve comprehensive properties of composites, modify numerous weakness of composites' properties and solve practical problems in engineering such as weak toughness and hardness required to be improved, simultaneously, so as to push wide applications of new kinds of composites in usages of main-standing parts.

Design and research development of all-on experiments to research POSS reinforced composites were induced to long research cycle and high cost which could not reach development speed of new-kinds of composites. However, the use of materials-on-computing methods such as molecular mechanical method and molecular dynamic method could achieve well design and development of advanced composites and the research cycle was greatly shortened. These methods have been the key path of researching and developing new materials. However, public literatures of predictive methods of physic and chemical properties of POSS/Polymer nanocomposites still remained in theoretical phase[8-14], not suitable for corresponding applications in materials research and development. Therefore, a design method convenient to practical engineering applications of POSS/resin matrix composites was built in this paper so as to provide technical support for practical applications of equipments.

\section{FOUNDATION OF ANALYSIS METHOD}

First, confirm that you have the correct template for your paper size. This template has been tailored for output on the US-letter paper size. If you are using A4-sized paper, please close this file and download the file for "MSW_A4_format". The rational relationships between macro-properties and corresponding properties, macro-properties and micro-structures were built, and the response rules and natures of POSS/resin composites' micro- and macro-structures under some certain conditions were revealed. All above could provide necessary theoretical basis and means for modified design, property evaluation of POSS/resin composites.

Compatibility between POSS and polymer was the key element of effecting whether POSS as nanofillers could improve mechanical properties of polymer, therefore, compatibility between POSS and resin could directly make effects on modification results of POSS to resin. Well compatibility between POSS and resin could make POSS uniformly spread in or into resin during polymerization and uniform phase system was formed so that resin system could be greatly improved. However, bad compatibility between POSS and resin induced into the separation of POSS and resin phases during polymerization, POSS would cluster in some places, materials defects would form, and modification effects would not be so good. Therefore, the modified-resin effects would be totally different with POSS blended into different resins.

Therefore, a new kind of multi-step effective modulus predictive method was built to predict effective modulus of POSS/resin composites so as to provide theoretical foundations for materials design and property of composites.

The procedures on predictive method of effective modulus of POSS/resin composites were as follows:

First, the following hypotheses were given according to the compatibility of POSS in different resins system for multiple engineering practical conditions. 
(1)Adding POSS into resin, it was hypothesized that a new kind of interface material which was used to describe compatibility between POSS and resin was formed between POSS and resin.

(2)Commonly, the compatibility between POSS and resin was not so perfect in practical conditions, and the interface compatibility was totally connected with manufacturing process. The elastic modulus of interface material was available defined according to practical engineering situations. That was, if compatibility between POSS and resin was well, it was hypothesized that elastic modulus of interface material between POSS and resin was totally close to elastic modulus of POSS, signed as $E_{i}$. However, if compatibility between POSS and resin was not so well, $E_{i}$ was discounted in ratio to the compatibility degree between POSS and resin.

(3) It was hypothesized that the weight ratio of interface material in the whole composite was signed as $P_{\text {coat }}$ and weight percentage of POSS in all composite was signed as $P_{\text {poss }}$, the relationship between $P_{\text {coat }}$ and $P_{\text {poss }}$ was as follows, and $C$ was experimental constant.

$$
P_{\text {coat }}=C P_{\text {poss }}
$$

However, during manufacturing procedure of POSS/resin composites, the whole-materials components or manufacturing methods under the same material-system adopted by researchers were different and therefore material-interface properties between POSS and resin were different. Therefore, according to practically different materials system and corresponding manufacturing processes, few experiments which only needed elastic modulus of one random content-ratio POSS/resin composites were made to predict effective elastic modulus of any other content ratio of POSS/resin composites under the same manufacturing processes by obtaining $C$ and $E_{i}$ based on numerical progressive iterative approximation method and compute the above two values as basic materials parameters so as to build the whole analysis model in the next step.

Computing procedures of effective modulus of POSS/resin composites were as follows.

(1) By making full use of Mori-Tanaka method[15] computational program in DIGIMAT software and elastic modulus of one random content ratio of POSS/resin composites' experimental result, experimental constant $C$ and interface elastic modulus $E_{i}$ by numerical progressive iterative approximation method were obtained as basis materials-input-parameters of this model to build the whole analysis model.

(2)Equivalent modulus of two-phase composites composed of POSS and interface material that were considered as one equivalent uniform reinforced material was computed by applying Mori-Tanaka method.
(3)The effective modulus of equivalent uniform reinforced resin material was computed by applying Mori-Tanaka method again.

\section{MODEL VALIDATIONS AND EXAMPLES ANALYSIS}

\section{A. Mechanical Property Parameters of POSS}

According to literature[17], mechanical property parameters of POSS were that elastic modulus was 8.11GPa and its Poisson ratio was 0.3 .

\section{B. Mechanical Property Parameters of Resins}

According to literature[16], mechanical property parameters of AFG-90/DDS (4,4'-Diaminodiphenyl sulfone) were that elastic modulus was $2.66 \mathrm{GPa}$ and its Poisson ratio was 0.3.

Mechanical property parameters of AFG-90/MeTHPA/TEA were that elastic modulus was $3.33 \mathrm{GPa}$ and its Poisson ratio was 0.3 .

\section{Effective Modulus Prediction and Experiment Validation of POSS Modified AFG-90/DDS}

Effective modulus of POSS Modified AFG-90/DDS was computed and analyzed based on method built in this project. Based on 10wt\% POSS/resin materials' elastic modulus experiment value $E=3.59 \mathrm{GPa}$, applying Mori-Tanaka computing procedure in DIGIMAT and numerical progressive iterative approximation method, interface material experiment constant $C=2$ in this model and interface material elastic modulus $E_{i}=8 \mathrm{GPa}$ were obtained. The above as input conditions of interface material's basis mechanical parameters, effective modulus of other content POSS/resin composites under the same manufacturing conditions were predicted. Effective modulus of $1 \mathrm{wt} \%, 3 \mathrm{wt} \%$ and $5 \mathrm{wt} \%$ POSS/resin composites were predicted and the comparisons were made between computing results and experimental results.

Comparisons between effective modulus predictive results and experimental results of POSS modified AFG-90/DDS by making full use of three methods, method built in this project, common Mori-tanaka method without considering interface and traditional mixture model method were made, shown in Table 1.

It was seen from comparison data results, the highest error of effective modulus between predictive result and experimental result by use of Mori-Tanaka without considering interface was up to $20.5 \%$ (predictive conformability was $79.5 \%$ and predictive error was huge while with high content POSS/resin composites). The highest error of effective modulus between predictive results and experimental results by use of traditional mixture model was up to $16.76 \%$ (predictive conformability was 83.24\% and predictive error was huge while with the same content POSS/resin composites). However, the highest error of effective modulus between predictive results and experimental results by use of method built in this project was up to $11.9 \%$, that means, predictive conformability was $88.1 \%$, greatly improved by Mori-Tanaka without considering interface and traditional mixture model. 
TABLE I. COMPARISONS OF EFFECTIVE MODULUS BETWEEN PREDICTION AND EXPERIMENT RESULTS OF POSS MODIFIED AFG-90/DDS

\begin{tabular}{|c|c|c|c|c|c|c|c|c|}
\hline \multirow[b]{2}{*}{ No. } & \multirow[b]{2}{*}{$\begin{array}{l}\text { POSS } \\
\text { content } \\
\text { (wt\%) }\end{array}$} & \multirow{2}{*}{$\begin{array}{c}\text { Experimental results } \\
\text { of effective modulus } \\
{[16]} \\
\text { (GPa) }\end{array}$} & \multicolumn{2}{|c|}{$\begin{array}{l}\text { Method built in this project } \\
\text { (Considering interface effect) }\end{array}$} & \multicolumn{2}{|c|}{$\begin{array}{l}\text { Common Mori-Tanaka Method } \\
\text { (Without considering } \\
\text { interface effect) }\end{array}$} & \multicolumn{2}{|c|}{ Traditional Mixture Model } \\
\hline & & & $\begin{array}{l}\text { Predictive results of } \\
\text { effective modulus } \\
\text { (GPa) }\end{array}$ & $\begin{array}{c}\text { Error } \\
(\%)\end{array}$ & $\begin{array}{l}\text { Predictive results } \\
\text { of effective } \\
\text { modulus } \\
\text { (GPa) }\end{array}$ & $\begin{array}{l}\text { Error } \\
(\%)\end{array}$ & $\begin{array}{l}\text { Predictive } \\
\text { results of } \\
\text { effective } \\
\text { modulus } \\
(\mathrm{GPa})\end{array}$ & $\begin{array}{l}\text { Error } \\
(\%)\end{array}$ \\
\hline 1 & 0 & 2.66 & - & - & - & - & - & - \\
\hline 3 & 3 & 2.80 & 2.91 & 3.9 & 2.74 & 2.1 & 2.82 & 0.71 \\
\hline 4 & 5 & 3.52 & 3.10 & 11.9 & 2.80 & 20.5 & 2.93 & 16.76 \\
\hline 5 & 10 & 3.59 & 3.61 & 0.6 & 2.95 & 17.8 & 3.21 & 10.59 \\
\hline
\end{tabular}

D. Effective Modulus Prediction and Experiment Validation of POSS Modified AFG-90/MeTHPA/TEA

Effective modulus of POSS Modified AFG-90/MeTHPA TEA was computed and analyzed based on method built in this project. Based on 10wt\% POSS/resin materials' elastic modulus experiment value $E=3.47 \mathrm{GPa}$, applying Mori-Tanaka computing procedure in DIGIMAT and numerical progressive iterative approximation method, interface material experiment constant $C=0.005$ in this model and interface material elastic modulus $E_{i}=0.03 \mathrm{GPa}$ were found. The above as input conditions of interface material basis mechanical parameters, effective modulus of other content POSS/resin composites under the same manufacturing situations were predicted. Effective modulus of $1 \mathrm{wt} \%$, 3wt\% and $5 \mathrm{wt} \%$ POSS/resin composites were predicted and the comparisons were made between computing results and experimental results.

Comparisons of effective modulus predictive results and experimental results of POSS modified AFG-90/MeTHPA /TEA by making full use of three methods, method built in this project, common Mori-tanaka method without considering interface and traditional mixture model method were made, shown in Table 2.

TABLE II. COMPARISONS OF EFFECTIVE MODULUS BETWEEN PREDICTION AND EXPERIMENT RESULTS OF POSS MODIFIED AFG-90/METHPA

\begin{tabular}{|c|c|c|c|c|c|c|c|c|}
\hline \multirow{2}{*}{ No. } & \multirow{2}{*}{$\begin{array}{l}\text { POSS } \\
\text { content } \\
\text { (wt\%) }\end{array}$} & \multirow{2}{*}{$\begin{array}{l}\text { Experimental results } \\
\text { of effective modulus } \\
{[16]} \\
\text { (GPa) }\end{array}$} & \multicolumn{2}{|c|}{$\begin{array}{l}\text { Method built in this project } \\
\text { (Considering interface } \\
\text { effect) }\end{array}$} & \multicolumn{2}{|c|}{$\begin{array}{c}\text { Common Mori-Tanaka Method } \\
\text { (Without considering } \\
\text { interface effect) }\end{array}$} & \multicolumn{2}{|c|}{ Traditional Mixture Model } \\
\hline & & & $\begin{array}{l}\text { Predictive results } \\
\text { of effective } \\
\text { modulus } \\
\text { (GPa) } \\
\end{array}$ & $\begin{array}{c}\text { Error } \\
(\%)\end{array}$ & $\begin{array}{l}\text { Predictive results } \\
\text { of effective } \\
\text { modulus } \\
(\mathrm{GPa}) \\
\end{array}$ & $\begin{array}{l}\text { Error } \\
(\%)\end{array}$ & $\begin{array}{l}\text { Predictive results } \\
\text { of effective } \\
\text { modulus } \\
(\mathrm{GPa}) \\
\end{array}$ & $\begin{array}{c}\text { Error } \\
(\%)\end{array}$ \\
\hline 1 & 0 & 3.33 & 3.33 & - & - & - & - & - \\
\hline 2 & 1 & 3.36 & 3.34 & 0.6 & 3.36 & 0 & 3.38 & 0.6 \\
\hline 3 & 3 & 3.39 & 3.37 & 0.6 & 3.42 & 0.9 & 3.47 & 2.4 \\
\hline 4 & 5 & 3.45 & 3.39 & 1.7 & 3.47 & 0.6 & 3.57 & 3.5 \\
\hline 5 & 10 & 3.47 & 3.46 & 0.3 & 3.62 & 4.3 & 3.81 & 9.8 \\
\hline
\end{tabular}

It was seen from comparison data results, the highest error of effective modulus between predictive result and experimental result by use of Mori-Tanaka without considering interface was up to $4.3 \%$. The highest error of effective modulus between predictive results and experimental results by use of traditional mixture model was up to $9.8 \%$ (predictive conformability was $83.24 \%$ and predictive error was huge while with the same content POSS/resin composites). However, the highest error of effective modulus between predictive results and experimental results by use of method built in this project was up to $1.7 \%$, greatly improved by Mori-Tanaka method and traditional mixture model method without considering interface's effect.
For POSS Modified AFG-90/DDS, effective modulus of resin after adding $10 \mathrm{wt} \%$ POSS was improved by $35 \%$ by comparing with net-resin. For POSS Modified AFG-90/MeTHPA/TEA, effective modulus of resin after adding $10 \mathrm{wt} \%$ POSS was improved by $4.2 \%$ by comparing with net-resin. It was seen that the compatibility of POSS in AFG-90/DDS was well. Well compatibility of POSS/resin could spread or insert into resin in forms of molecular uniformly and uniform phase was formed, which made mechanical properties of resin materials system greatly improved. 


\section{CONCLUSIONS}

(1) A new multi-step effective modulus predictive method of POSS/resin composite was built according to practical engineering conditions.

(2)The effective modulus predictive results and experimental results for POSS reinforced AFG-90/DDS and POSS Modified AFG-90/MeTHPA/TEA with different content ratios of POSS in resin systems by making use of effective modulus predictive method built in this project were compared and analyzed. The results showed that the maximal error of predictive result was up to $11.9 \%$, and that means, the predictive precision was above $88.1 \%$. The predictive results were well and it could provide theoretical basis for material design and property evaluation of POSS/resin composites. However, the currency of Common Mori-Tanaka Method and traditional mixture model was not well, and predictive results were just well for latter while large error for AFG-90/DDS materials' system.

\section{REFERENCES}

[1] J. Pyun, K. Matyjaszewski, J. Wu, et al, ABA Triblock Copolymers Containing Polyhedral Oligomeric Silsesquioxane Pendant Groups, Synthesis and Unique Properties, Polymer, 44, 2739-2750.

[2] R. Verker, E. Grossman, N. Eliaz, Erosion of POSS-polyimide Films Under Hypervelocity Impact and Atomic Oxygen, The Role of Mechanical Properties at Elevated Temperatures, Acta Materialia, 2009, 57(4), 1112-1119.

[3] H.U. Kim, Y.H. Bang, S.M. Choi, et al, Morphology and Mechanical Properties of PET by Incorporation of Amine-polyhedral Oligomeric Silsesquioxane, Composites Science and Technology, 2008, 68(13), 2739-2747.

[4] R.J. Yang, W.C. Zhang, X.M. Li, et al, Mechanical and Thermal Properties and Flame Retarancy of Phosphorus-containing Polyhedral Oligomeric Silsesquioxane(DOPO-POSS)/Polycarbonate Composites, Polymer Degradation and Stability, 2010, 95(12), 2541-2546.

[5] Z.P. Zhang, A.J. Gu, G.Z. Liang, et al, Thermo- oxygen Degradation Mechanisms of POSS/Epoxy Nano- composites, Polymer Degradation and Stability, 2007, 92: 1986-1993.

[6] A. Buchman, H. Dodiuk-Kenig, S. Kenig, Improved Fracture Toughness of Epoxy Adhesives Filled with POSS Nanoparticles, WCARP III, 2006, 58-63.

[7] Y.J. Sheng, W.J. Lin, W.C. Chen. Network Structures of Polyhedral Oligomeric Silsesquioxane Based Nanocomposites: A Monte Carlo study, The Journal of Chemical Physics. 2004, 121(19), 9693-9701.

[8] M.H. Lamm, T. Chen, S.C. Glotzer. Simulated Assembly of Nanostructured Organic/inorganic Networks, Nano Letters, 2003, 3(8), 989-994.

[9] F.L. Zeng, Mechanical Models and Properties Characterization to the POSS Organic-inorganic Nano Hybrid Materials, Harbin Institute of Technology. 2006

[10] Y. Sun, F.L. Zeng, Molecule Mechanics Simulation on the Deformation and Damage Process in POSS Nanocomposite, Advances in Fracture and Damage Mechanics VI, 2007, 348-349: 109-112.

[11] E.L Hu, Deformation Mechanism and Meso-micro Mechanical Model of PE/POSS Nano Hybrid Materials, Dissertation for the Doctoral Degree in Engineering, Harbin Institute of Technology, 2012.

[12] J.J. Schwab, J.D. Lichtenhan, Polyhedral Oligomeric Silsesquioxanes (POSS): Silicon Based Monomers and Their Use in The Preparation of Hybrid Polyurethanes, Mater Res Soc Symp Proc, 1998, 519, 21-27.

[13] R.K. Bharadwaj, R.J. Berry, B.L. Farmer, Molecular Dynamics Simulation Study of Norbornene-POSS Polymers, Polymer, 2000, 41: 7209-7221.

[14] S. Bizet, J. Galy, J.F. Gerard, Molecular Dynamics Simulation of Organic-inorganic Copolymers Based on Methacryl-POSS and Methyl
Methacrylate, Polymer, 2006, 47, 8219-8227.

[15] T. Mori, K. Tanaka. Average Stress in Matrix and Average Energy of Materials with Misfitting Inclusions. Act. Metall., 1973, 21, 571-574.

[16] Q.Y. Qiu, Research on Thermal and Chemo-rheology Investigations of POSS Modified Epoxy Resin, National University of Defense Technology, 2007.

[17] J. Li, Molecular Dynamics Simulations and Mechanical Properties of POSS Nano Hybrid Materials. Dissertation for the Doctoral Degree in Engineering, Harbin Institute of Technology, 2011. 